\title{
La recherche économique spatiale, urbaine et régionale au Québec : réflexion sur les 35 dernières années et sur l'avenir ${ }^{1}$
}

\author{
Luc-Normand Tellier \\ Université du Québec à Montréal
}

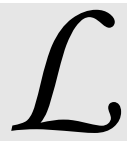
a fondation de l'INRS-Urbanisation en 1970 a marqué une date importante dans l'évolution de la recherche économique spatiale, urbaine et régionale au Québec. Trente-cinq ans plus tard, il est utile de tenter de dresser un bilan critique de l'évolution de cette recherche dans l'ensemble du Québec. Je le ferai d'un point de vue très personnel en renonçant à l'exhaustivité et en évitant de porter un jugement sur les contributions des uns et des autres. Mon propos ne se limitera nullement à la place de l'INRS-Urbanisation dans la recherche économique spatiale, urbaine et régionale au Québec.

Ce centre de recherche n'est à mes yeux ni l'alpha, ni l'oméga, ni même le coeur de ce champ disciplinaire au Québec. Cependant, sa création a correspondu à un effort conscient d'asseoir cette discipline sur des assises solides, c'està-dire sur une équipe d'économistes urbains et régionaux, sur un programme de recherche à orienta-

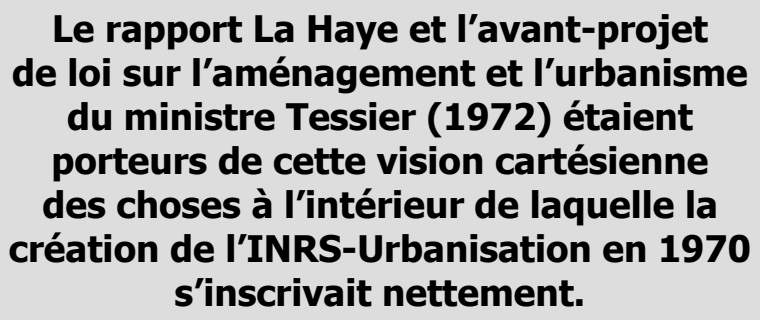

Le rapport La Haye et l'avant-projet de loi sur l'aménagement et l'urbanisme du ministre Tessier (1972) étaient porteurs de cette vision cartésienne des choses à l'intérieur de laquelle la création de I'INRS-Urbanisation en 1970 s'inscrivait nettement.

Lors de sa création, l'INRS-Urbanisation s'est inscrit dans le courant dominant issu de la Révolution tranquille suivant lequel l'État du Québec et, incidemment, l'aménagement du territoire devaient se structurer à partir de l'idée de planification économique importée du modèle français (marqué par l'existence d'un ministère du Plan). Cet idéal (incarné en bonne partie par Roland Parenteau) a donné lieu en 19601961 à la constitution du Conseil d'orientation économique (dissous en 1968), à la mise sur pied, en 1963, du Bureau de l'aménagement de l'Est du Québec (BAEQ) et à la création en 1968 de l'Office de planification du Québec, devenu l'Office de planification et de développement du Québec (OPDQ) en 1969. Ces organismes visaient à établir une cohérence verticale entre la planification économique provinciale et l'aménagement tion économique et sur l'établissement d'une complémentarité systématique avec une équipe et une approche plus sociologiques devant constituer la seconde « mamelle» de l'INRS-Urbanisation (si je puis emprunter cette expression à Sully).

J'ai moi-même été témoin de cet effort, d'abord à titre d'assistant de recherche dès la fondation de l'INRS, puis à titre de premier boursier de cette institution qui a financé mon doctorat en science régionale obtenu à l'Université de Pennsylvanie en 1973 et, enfin, à titre de directeur de l'INRS-Urbanisation pendant deux ans et demi, de 1981 à 1983. local et régional, ainsi qu'une cohérence horizontale entre toutes les initiatives de planification locales grâce à l'imposition d'un cadre provincial dominé par une logique avant tout économique. Le rapport $\mathrm{La}$ Haye $^{2}$ et l'avant-projet de loi sur l'aménagement et l'urbanisme du ministre Tessier (1972) étaient porteurs de cette vision cartésienne des choses à l'intérieur de laquelle la création de l'INRS-Urbanisation en 1970 s'inscrivait nettement.

Entre 1970-1972 et 1979 (date de la loi 125 sur l'aménagement et l'urbanisme) s'est opéré le divorce historique entre les idéaux de planification économique et 
d'aménagement du territoire. Un ouvrage collectif dirigé par le regretté Jacques Léveillée ${ }^{3}$ fait l'autopsie du rêve abandonné. J'y écris que la " prudence manifestée $[\ldots]$ à l'endroit du système économique prédominant en Amérique du Nord [où l'idéal d'une planification économique n'a jamais vraiment existé] a conduit le gouvernement québécois à distinguer de plus en plus, puis à séparer clairement les notions de planification économique et d'aménagement. Le lien entre économie et environnement s'estompant, on semble avoir eu tendance à privilégier une planification de l'espace à caractère statique, détachée du processus budgétaire et extérieurement ouverte à un haut degré de participation sans que cela débouche forcément, par contre, sur une véritable concertation ni sur une mobilisation des forces économiques et sociales $»^{4}$.

Ce divorce entre l'idée de planification économique et celle d'aménagement du territoire portait en germe la marginalisation de l'économie spatiale, urbaine et régionale, cette dernière étant par vocation le lien théorique principal devant unir planification économique et aménagement du territoire. Nous verrons que la suite de l'histoire n'a fait que confirmer ce qui aurait pu être prévu alors.

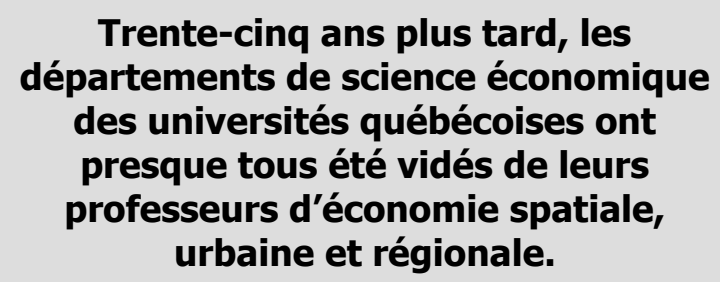

\section{Le divorce de l'économie urbaine et régionale et de l'économie classique}

L'expérience du BAEQ avait fortement été inspirée par la théorie économique des pôles de croissance, bien que les sociologues de l'Université Laval aient eu une présence marquée aux côtés des économistes de la même université dans ce projet. Le célèbre rapport Higgins, Martin, Raynault ${ }^{5}$, rédigé par trois économistes «classiques » de 1'Université de Montréal, ébranla les colonnes du temple idéologique qui avait abrité l'expérience du BAEQ en se servant de la même théorie des pôles de croissance. Ses auteurs lançaient un cri d'alarme en soulignant qu'en mettant trop l'accent sur le développement de ses régions marginales, le Québec compromettait les chances de la région de Montréal de maintenir sa place dans l'économie canadienne face à Toronto.

Ce rapport a eu et a encore un énorme retentissement. Il contribua à discréditer la théorie des pôles de croissance (dont Higgins, Martin et Raynault s'inspiraient pourtant) en montrant qu'on pouvait lui faire dire tout et son contraire. Il discrédita les économistes dans le domaine du développement local et régional (comment se fier à des experts dont les théories peuvent à la fois encourager et décourager les efforts de développement local ?). Enfin, il contribua à marginaliser l'économie spatiale, urbaine et régionale à l'intérieur des départements de science économique du Québec.

En effet, le caractère impressionniste, littéraire et peu mathématique de la théorie des pôles de croissance ainsi que son absence de bases théoriques, microéconomiques ou macro-économiques solides discréditèrent, aux yeux de très nombreux économistes « classiques ", tout un champ disciplinaire, celui de l'économie spatiale, urbaine et régionale auquel ils n'avaient, le plus souvent, jamais vraiment été initiés. Trente-cinq ans plus tard, les départements de science économique des universités québécoises ont presque tous été vidés de leurs professeurs d'économie spatiale, urbaine et régionale ${ }^{6}$, et le divorce entre ce champ disciplinaire et l'économie classique est pratiquement consommé. Il faut noter qu'aux États-Unis, la même évolution s'est produite, d'abord avec la dissociation de la «Regional Science» et de la science économique, puis avec la quasi-disparition de la « Regional Science » comme discipline autonome.

\section{Le divorce de l'économie spatiale et de l'économie urbaine et régionale}

Le divorce de la science économique classique et de l'économie spatiale, urbaine et régionale (appelée $R e$ gional Science aux États-Unis et un peu ailleurs) annonça l'éclatement de cette dernière en une multitude de courants dont les quatre principaux me semblent être :

1. L'économie spatiale proprement dite, profondément déductive (tout comme l'économie classique d'ailleurs), à formulation et à raisonnement mathématiques, trop souvent ésotérique et difficilement compréhensible pour le commun des mortels; l'é- 
conomie des transports doit être vue comme un prolongement de l'économie spatiale; elle partage avec cette dernière son caractère quantitatif et mathématique.

2. L'économie urbaine et régionale «descriptionniste » utilisant à fond les quatre opérations élémentaires de l'arithmétique (l'addition, la soustraction, la multiplication et la division) pour décrire le monde économique urbain et régional sans recourir à quelque modèle mathématique que ce soit, ni même à l'économétrie; ce courant table exclusivement sur la description du passé et du présent, sur le benchmarking et sur la collecte des données les plus récentes et les plus exclusives; il renonce à l'avance à formuler quelle que théorie que ce soit et à produire méthodiquement quelle que projection que ce soit; pour lui, décrire et comparer constituent l'alpha et l'oméga de l'économie urbaine et régionale.

3. L'économie urbaine et régionale «synthétisante » mettant à profit l'observation des variables économiques, mais aussi des variables socioculturelles dans le dessein de formuler des théories non mathématiques basées sur la théorie économique traditionnelle, mais aussi sur des disciplines aussi variées que la sociologie, la psychologie, la théorie des organisations, la science politique, etc.; ce courant est marqué par un souci constant de synthèse et d'analyse du développement urbain et régional dans une perspective multidisciplinaire, mais dans une optique économique; le livre de Proul $x^{7}$ incarne parfaitement ce courant.

4. L'école du développement local qui a, en grande partie, rompu tout lien avec la science économique classique et l'économie spatiale.

Le divorce entre l'économie spatiale et l'économie urbaine et régionale «descriptionniste » me semble profond. Il correspond aux clivages qui existent entre l'arithmétique et l'algèbre, entre l'arithmétique et le raisonnement mathématique, entre l'idée de système et celle de recension journalistique des faits, entre la modélisation et la simple description ainsi qu'entre la théorie et la simple compilation de données.

Par contre, le lien demeure entre l'économie spatiale et l'économie urbaine et régionale "synthétisante », bien que le dialogue entre les deux devienne de plus en plus difficile au fur et à mesure que le niveau d'ésotérisme mathématique de l'économie spatiale augmente. Si ce lien demeure, il s'affaiblit sans doute, ce qui fait craindre le pire. Il est plus que vraisemblable que la faute en incombe beaucoup plus aux économistes "spatiaux » qu'aux économistes urbains et régionaux "synthétisants », ces derniers faisant des efforts louables d'intégration des concepts de l'économie spatiale, alors que les économistes spatiaux perdent en étendue ce qu'ils gagnent en approfondissement de leur démarche. Si les premiers trop embrassent et mal étreignent, les seconds trop étreignent et mal embrassent.

\section{Le divorce entre l'économie spatiale et l'économie urbaine et régionale « descriptionniste » me semble profond.}

Ce qui fait craindre le pire pour l'avenir du lien qui unit toujours l'économie spatiale et l'économie urbaine et régionale "synthétisante », ce n'est pas la volonté des uns et des autres de maintenir les ponts ouverts. C'est plutôt l'évolution divergente des langages, de plus en plus mathématique dans le cas des premiers et de plus en plus littéraire et même sociologique dans le cas des seconds, mais c'est aussi la perte de contact avec le monde réel de plusieurs économistes «spatiaux» et le souci, toujours réaffirmé, de pertinence des "synthétisants ». Il y a là comme un dialogue de malentendants devenant de plus en plus sourds.

Pour ce qui est de l'école du développement local, elle vole de ses propres ailes loin de l'économétrie, des méthodes économiques d'évaluation de projets, de la théorie économique spatiale et des cadres classiques de la microéconomie et de la macroéconomie. Les seuls concepts économiques qui la lient encore à l'économie sont ceux d'économies externes et d'agglomération.

\section{Concevoir le développement loin de la science économique}

Ces multiples divorces entre économistes seraient de peu de conséquences s'ils ne conduisaient à une aberration, soit à aborder de plus en plus le développement loin de la science économique. J'ai eu à donner plusieurs cours et j'ai écrit un livre sur les méthodes 
économiques d'évaluation de projets publics. J'ai donné ces cours au Québec dans le cadre d'une mâ̂trise interdisciplinaire en études urbaines, puis en Haïti au Centre de techniques de planification et d'économie appliquée (CTPEA) et en Guadeloupe dans le cadre d'un DESS (diplôme d'études supérieures spécialisées) en ingénierie économique du développement et de l'environnement. Ces cours furent tout à fait appréciés par les étudiants d'Haïti et de la Guadeloupe qui avaient une formation en science économique, alors qu'ils ont soulevé tant de critiques de la part des étudiants québécois ayant peu ou pas de notions économiques qu'ils ont dû être réservés à une petite minorité.

Nous assistons présentement dans les universités québécoises à une évolution où les économistes classiques s'intéressent de moins en moins au développement urbain et régional, alors que ceux qui sont fascinés par ce développement tournent de plus en plus le dos à la science économique, aux méthodes économétriques et aux méthodes économiques d'évaluation de projet. Où cela conduit-il ? Je pose la question tout en disant qu'à mes yeux, quelque chose ne va pas dans tout cela.

Je me demande si l'erreur fondamentale n'a pas été de tenter de plonger l'économie spatiale, urbaine et régionale dans un bain trop multidisciplinaire, comme on l'a fait à l'INRS-Urbanisation et comme je l'ai fait moi-même dans le département d'études urbaines et touristiques que j'ai fondé et dont j'aurai été le directeur pendant treize ans. Cette approche a contribué, plus que tout autre facteur, à favoriser les divorces dont j'ai parlé et à noyer la science économique et l'approche quantitative dans un bassin trop souvent allergique à l'économique et au quantitatif.

Mes expériences d'enseignement en Guadeloupe et à Haïti me démontrent que d'autres voies existent et qu'il est possible de faire fleurir l'économie spatiale, urbaine et régionale dans un esprit d'ouverture aux autres disciplines pourvu qu'il soit admis que le point de départ est l'économie et que tous les étudiants et tous les professeurs doivent avoir une base solide dans cette discipline.

Hélas, nulle part au Québec cette approche n'a été privilégiée, ce qui a conduit à marginaliser totalement l'économie spatiale, urbaine et régionale.
Je me demande si l'erreur fondamentale n'a pas été de tenter de plonger l'économie spatiale, urbaine et régionale dans un bain trop multidisciplinaire.

\section{Le divorce du savoir et du savoir-faire économiques urbains et régionaux}

Comme me l'a fait remarquer Marc-Urbain Proulx, l'évolution que je viens de décrire a fortement contribué à un autre divorce : celui du savoir et du savoirfaire économiques urbains et régionaux. Actuellement, les responsables du développement économique régional n'ont accès à pratiquement aucun diplômé initié à l'économie spatiale, urbaine et régionale qui soit capable de faire des analyses avantages-coûts, des calculs de rentabilité fiscale, des calculs de rentabilité, des analyses input-output et de concevoir des modèles mathématiques économétriques ou autres dans les règles de l'art. Les diplômés qui ont des notions d'économie spatiale, urbaine et régionale sont incapables d'accomplir de telles tâches, et les diplômés qui peuvent les accomplir ne connaissent rien à l'économie spatiale, urbaine et régionale. Trente-cinq ans après la fondation de l'INRS-Urbanisation, nous en sommes-là.

Il est possible que les théoriciens de l'économie spatiale, urbaine et régionale aient failli à la tâche et aient négligé d'alimenter le savoir-faire local. Je crois qu'ils ont leur part de responsabilités, mais que l'option universitaire retenue, celle de la multidisciplinarité à tout prix, a été mauvaise. Selon mon expérience, l'approche quantitative ne peut survivre dans un milieu où les qualitatifs dominent. Elle ne peut s'épanouir qu'en zone réservée, ce qui ne veut absolument pas dire que les quantitatifs doivent se fermer aux approches qualitatives. Au contraire, je crois que les quantitatifs s'ouvriront d'autant plus facilement au qualitatif qu'ils se sentiront sûrs d'eux-mêmes et non menacés. J'ai très souvent vu des qualitatifs être très agressifs à l'endroit des quantitatifs. J'ai rarement vu l'inverse.

\section{Que faire face à tout cela ?}

J'ose proposer un virage majeur, celui de la création au Québec d'un programme de maîtrise en économie spatiale, urbaine et régionale auquel seraient associés 
des départements de science économique classique et des économistes spatiaux, urbains et régionaux issus de divers établissements universitaires. Ce programme pourrait éventuellement déboucher sur un doctorat en économie spatiale, urbaine et régionale. Les deux programmes devraient couvrir les domaines de l'économie spatiale (à formulation mathématique), de l'économie des transports, de l'économie urbaine et régionale (au sens «littéraire » du terme), de l'analyse statistique spatiale, de l'économétrie spatiale, de la modélisation spatiale et des méthodes économiques d'évaluation de projet.

Cependant, ce virage pédagogique devrait être assorti d'un virage sur le plan de la recherche. Ce dernier impliquerait que soient renoués les liens entre l'économie non spatiale (telle qu'enseignée dans les départements de science économique) et l'économie spatiale, urbaine et régionale. Cela fait, je crois qu'il faudrait reprendre presque à zéro l'étude des économies d'agglomération à la lumière de la théorie de la localisation ${ }^{8}$. Il faudrait aussi accorder à l'avenir au moins autant d'importance à l'analyse des flux (investissements, commerce, migration) qu'à l'analyse des stocks (population, production), ce qui n'a pas vraiment été fait jusqu'ici. Enfin, il faudrait envisager la possibilité de repenser la concurrence spatiale dans un contexte marqué par les trajectoires topodynamiques ${ }^{9}$.

\section{Notes biographiques}

Luc-Normand Tellier est directeur du Département d'études urbaines et touristiques de l'UQAM. Il vient de publier aux Éditions LIBER un ouvrage de 580 pages intitulé « Redécouvrir l'HISTOIRE MONDIALE, sa dynamique économique, ses villes et sa géographie ».

\section{Notes et références}

1 Communication présentée dans le cadre de l'ACFAS, le 10 mai 2005 à l'Université du Québec à Chicoutimi.

2 La Haye, J.-C. et al. (1968). Rapport de la Commission provinciale d'urbanisme, Gouvernement du Québec.

3 Léveillée, J. (dir.) (1982). L'aménagement du territoire au Québec: du rêve au compromis, Montréal, Nouvelle optique.

4 Tellier, L.-N. (1982). «Les dimensions économiques de l'aménagement du territoire au Québec », dans J. Léveillée, op. cit., p. 59-60.

5 Higgins, B., F. Martin et A. Raynault (1970). Les orientations du développement économique régional dans la province de Québec, Ottawa, Ministère de l'Expansion économique régionale.

6 Fernand Martin tient toujours le «fort » à l'Université de Montréal où il enseigne, au moins, depuis le début des années 1960. Il est le co-auteur d'un rapport récent qui fait, entre autres, l'historique des politiques régionales au Québec et au Canada (voir Joanis, M., F. Martin et S. St-Cerny (2004), Quel avenir pour les politiques de développement régional au Québec?, Montréal, CIRANO (Centre interuniversitaire de recherche en analyse des organisations).

7 Proulx, M.-U. (2002) L'économie des territoires au Québec : aménagement, gestion, développement, Québec, Presses de l'Université du Québec.

8 Tellier, L.-N. (2004). «Et si les économies d'agglomération n'existaient pas, notre monde serait-il différent? », Organisations et territoires, vol. 13, ${ }^{\circ} 3$, septembre 2004, p. $77-80$.

9 Tellier, L.-N. (2005). Redécouvrir l'histoire mondiale, sa dynamique économique, ses villes et sa géographie, Montréal, Éditions LIBER, 582 p. 


\section{Publicité}

\section{Doctorat en développement régional}

